\title{
The JEFF evaluated nuclear data project
}

A.J. Koning 1,a , M. Avrigeanu ${ }^{2}$, V. Avrigeanu ${ }^{2}$, P. Batistoni ${ }^{3}$, E. Bauge ${ }^{4}$, M.-M. Bé ${ }^{5}$, P. Bem ${ }^{6}$, D. Bernard ${ }^{7}$, O. Bersillon ${ }^{4}$, A. Bidaud ${ }^{8}$, O. Bouland ${ }^{7}$, A. Courcelle ${ }^{7}$, C.J. Dean ${ }^{9}$, P. Dos-Santos-Uzarralde ${ }^{4}$, B. Duchemin ${ }^{5}$, I. Duhamel ${ }^{10}$, M.C. Duijvestijn ${ }^{1}$, E. Dupont ${ }^{7,11}$, U. Fischer ${ }^{12}$, R.A. Forrest ${ }^{13}$, F. Gunsing ${ }^{11}$, W. Haeck ${ }^{14}$, H. Henriksson ${ }^{15}$, A. Hogenbirk ${ }^{1}$, T.D. Huynh ${ }^{5}$, R. Jacqmin ${ }^{7}$, C. Jouanne ${ }^{5}$, J. Keinert ${ }^{16}$, M.A. Kellett ${ }^{17}$, I. Kodeli ${ }^{15}$, J. Kopecky ${ }^{18}$, H. Leeb ${ }^{19}$, D. Leichtle ${ }^{12}$, J. Leppanen ${ }^{20}$, O. Litaize ${ }^{7}$, M.J. Lopez Jimenez ${ }^{4}$, M. Mattes ${ }^{16}$, E. Menapace ${ }^{21}$, R.W. Mills ${ }^{22}$, B. Morillon ${ }^{4}$, C. Mounier ${ }^{5}$, A.L. Nichols ${ }^{17}$, G. Noguere ${ }^{7}$, C. Nordborg ${ }^{15}$, A. Nouri ${ }^{10}$, R.L. Perel ${ }^{23}$, P. Pereslavtsev ${ }^{12}$, R.J. Perry ${ }^{9}$, M. Pescarini2 ${ }^{21}$, M. Pillon ${ }^{3}$, A.J.M. Plompen ${ }^{24}$, D. Ridikas ${ }^{11}$, P. Romain ${ }^{4}$, Y. Rugama ${ }^{15}$, P. Rullhusen ${ }^{24}$, C. de Saint Jean ${ }^{7}$, A. Santamarina ${ }^{7}$, E. Sartori ${ }^{15}$, K. Seidel ${ }^{25}$, O. Serot ${ }^{7}$, S. Simakov ${ }^{12}$, J.-Ch. Sublet ${ }^{7}$, S. Tagesen ${ }^{26}$, A. Trkov ${ }^{27}$, S.C. van der Marck ${ }^{1}$, and H. Vonach ${ }^{26}$

1 Nuclear Research and Consultancy Group NRG, P.O. Box 25, 1755 ZG Petten, The Netherlands

2 National Institute for Physics and Nuclear Engineering "Horia Hulubei”, P.O. Box MG-6, 76900 Bucharest-Magurele, Romania

3 ENEA Frascati, Fusion Department, Via Enrico Fermi 45, 00044 Frascati, Italy

${ }^{4}$ Commissariat à l'Énergie Atomique, DAM/DIF/DPTA, BP. 12, 91680 Bruyères-le-Châtel, France

5 CEA LIST/LNHB, C.E. Saclay, 91191 Gif-sur-Yvette, France

6 Academy of Sciences of the Czech Republic, Nuclear Physics Institute, 25068 Rez, Czech Republic

7 CEA Cadarache, DEN/DER/SPRC, 13108 Saint-Paul-lez-Durance, France

8 INPG/Grenoble Univ., Lab. de Physique Subatomique et Cosmologie (CNRS/IN2P3), Av. des Martyrs, 38026 Grenoble Cedex, France

9 Serco Assurance, Winfrith Technology Centre, Dorchester, Dorset, DT2 8DH, UK

${ }^{10}$ IRSN, BP. 92262, Fontenay-aux-Roses Cedex, France

11 CEA Saclay, DSM/Dapnia/SPhN, 91191 Gif-sur-Yvette, France

12 Forschungszentrum Karlsruhe, Institut für Reaktorsicherheit, Postfach 3640, 76021 Karlsruhe, Germany

13 UKAEA/EURATOM Fusion Association, Culham Science Centre, Abingdon, Oxfordshire, OX14 3DB, UK

14 SCK-CEN, Boeretang 200, 2400 Mol, Belgium

15 OECD Nuclear Energy Agency, 12, bd des Îles, 92130 Issy-les-Moulineaux, France

16 Universität Stuttgart, Institut für Kernenergetik und Energiesysteme, Pfaffenwaldring 31, 70550 Stuttgart, Germany

17 Nuclear Data Section, International Atomic Energy Agency, Wagramer Strasse 5, 1400 Vienna, Austria

18 JUKO Research, Kalmanstraat 4, 1817 HX Alkmaar, The Netherlands

19 Technische Universität Wien, Wiedner Hauptstrasse 8-10, 1040 Wien, Austria

20 TT Technical Research Centre of Finland, Lämpömiehenkuja 3 Espoo, P.O. Box 1000, 02044 VTT, Finland

${ }^{21}$ ENEA-Centro Ricerche "E. Clementel” FIS-NUC, Via Martiri di Monte Sole 4, 40129 Bologna, Italy

22 Nexia Solutions Ltd., B170 Sellafield Works, Seascale, Cumbria, CA20 1PG, UK

${ }^{23}$ Racah Institute of Physics, The Hebrew University of Jerusalem, 91904 Jerusalem, Israel

24 EC-JRC-IRMM, Retieseweg, 2440 Geel, Belgium

25 Technische Univ. Dresden, Institut für Kern- und Teilchenphysik, Mommsenstrasse 13, 01062 Dresden, Germany

${ }^{26}$ Inst. für Isotopenforschung und Kernphysik der Univ. Wien, Wäringerstr. 17/607, 1090 Wien, Austria

27 Institute Jozef Stefan, Jamova 39, 1000 Ljubljana, Slovenia

\begin{abstract}
The status of the Joint Evaluated Fission and Fusion file (JEFF) is described. JEFF-3.1 comprises a significant update of actinide evaluations, materials evaluations that have emerged from various European nuclear data projects, the activation library JEFF-3.1/A, the decay data and fission yield sub-libraries, and fusion-related data files from the EFF project. The revisions were motivated by the availability of new measurements, modelling capabilities and trends from integral experiments. Validations have been performed, mainly for criticality, reactivity temperature coefficients, fuel inventory and shielding of thermal and fast systems. Compared with earlier releases, JEFF-3.1 provides improved performance with respect to a variety of scientific and industrial applications. Following on from the public release of JEFF-3.1, the French nuclear power industry has selected this suite of nuclear applications libraries for inclusion in their production codes.
\end{abstract}

\section{Introduction}

The JEFF project involves evaluation efforts that cover the main nuclear data needs in the fields of fission and fusion applications. This initiative has provided the means for co-operative activities between participating countries while

${ }^{a}$ Presenting author, e-mail: koning@nrg-nl .com ensuring the most rational and efficient use of available resources. Although JEFF is an OECD/NEA project, the USA and Japan continue to develop their own nuclear data libraries, and therefore the JEFF project involves essentially scientists from European countries. The overall structure of the project is depicted in figure 1. Development of the JEFF libraries is not directly financed, and is based on the voluntary participation of European institutes and scientists. Staff at the NEA Data 


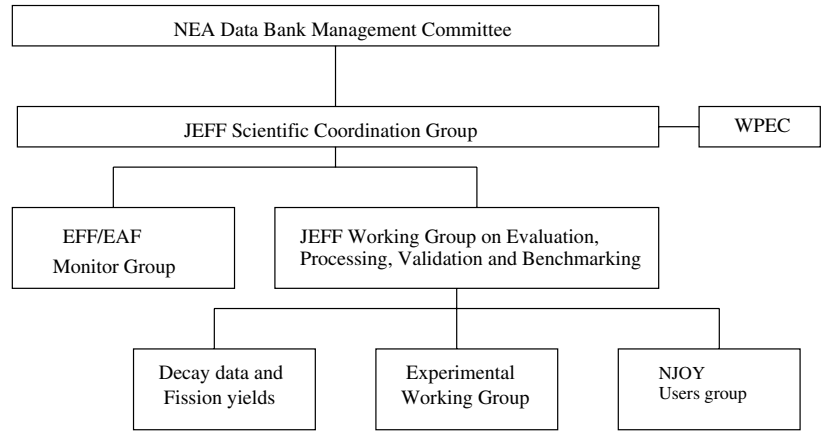

Fig. 1. Organisation of the JEFF project.

Bank ensure the maintenance of the JEFF library, and biannual JEFF meetings bring together experts in different areas such as data evaluations, experimental studies, reaction modelling, verification and compilation of the data under strict quality assurance procedures, file processing and benchmarking. The latest version of the JEFF-3.1 library was released in May 2005 [1], and contains a neutron-induced reaction data library with 381 isotopes or elements, a proton library with 26 isotopes, and thermal scattering law data for 9 materials. Special purpose libraries on radioactive decay data with 3852 isotopes and spontaneous and neutron fission yield data (for 19 isotopes) are available, as well as an activation data library containing 12617 neutron-induced reactions on 774 target nuclei, which is based on the European Activation File EAF2003. While existing reactors and fuel cycles remain the areas of essential application for JEFF, innovative reactor concepts (GEN-IV systems), transmutation of radioactive waste, fusion, medical applications, and various non-energy related industrial applications also make use of the JEFF data files. The European Fusion File (EFF) project contributes to this initiative through specific tasks on nuclear data evaluations, library production, verification of data, development of computer programs and validation of nuclear data through integral experiments.

\section{Status of JEFF-3.1}

There were various reasons for the upgrade from JEFF-3.0 to JEFF-3.1: all general and special purpose libraries were significantly improved and extended from 2002 to 2005. Moreover, following the release of the JEFF-3.0 general purpose file, various benchmark tests confirmed the expected performance improvements over JEF-2.2, but also indicated that the reactivities of low-enriched uranium (LEU) systems were underestimated by about $500 \mathrm{pcm}$. There were also other indications from deterministic and Monte Carlo analyses of reactor neutronics and fuel cycle issues, as well as activation and shielding problems for fusion, that significant improvements could be implemented. This situation led to JEFF-3.1, which is now judged by the JEFF community to be a more reliable basis for technological applications. JEFF-3.1 consists of the following sub-libraries:

- neutron general purpose library,

- neutron activation library,
Table 1. New neutron evaluations produced in 2002-2005.

\begin{tabular}{ll}
\hline Nuclei & Energy range and origin \\
\hline${ }^{40,42,43,44,46,48} \mathrm{Ca}$ & $0-200 \mathrm{MeV}$, NRG [2] \\
${ }^{45} \mathrm{Sc}$ & $0-200 \mathrm{MeV}, \mathrm{NRG}[2]$ \\
${ }^{46-50} \mathrm{Ti}$ & $0-20 \mathrm{MeV}$, IRK Vienna [3] \\
${ }^{54,56,57,58} \mathrm{Fe}$ & $0-200 \mathrm{MeV}, \mathrm{NRG}[4]$ \\
${ }^{70,72,73,74,76} \mathrm{Ge}$ & $0-200 \mathrm{MeV}, \mathrm{NRG}[5]$ \\
${ }^{99} \mathrm{Tc}$ & $0-200 \mathrm{MeV}, \mathrm{CEA} /$ Saclay/Cadarache, NRG [6] \\
${ }^{103} \mathrm{Rh}$ & $0-30 \mathrm{MeV}, \mathrm{CEA} /$ Cadarache/BRC [7] \\
${ }^{127,129} \mathrm{I}$ & $0-30 \mathrm{MeV}, \mathrm{CEA} /$ Cadarache/BRC [8] \\
${ }^{174-180} \mathrm{Hf}$ & $0-20 \mathrm{MeV}, \mathrm{CEA} /$ Cadarache [9] \\
${ }^{204,206,207,208} \mathrm{~Pb}$ & $0-200 \mathrm{MeV}, \mathrm{NRG}[10]$ \\
${ }^{209} \mathrm{Bi}$ & $0-200 \mathrm{MeV}, \mathrm{NRG}$ [10] \\
${ }^{236,237} \mathrm{U}$ & $0-30 \mathrm{MeV}, \mathrm{CEA} / \mathrm{BRC}$ \\
${ }^{238} \mathrm{U}$ & $0-30 \mathrm{MeV}, \mathrm{CEA} /$ Cadarache/BRC, IAEA [11-13] \\
${ }^{241} \mathrm{Am}$ & thermal only, CEA/Cadarache [14] \\
\hline
\end{tabular}

- thermal scattering law library,

- decay data library,

- fission yield data library,

- proton special purpose library.

We outline the most important developments below.

\subsection{Neutron general purpose library}

An important reason to develop the JEFF-3.1 neutron general purpose library is that a significant number of isotopes have undergone major revisions in recent years. The new neutron files produced in 2002-2005 are shown in table 1. All data libraries available in the world were critically examined for other isotopes to determine whether any evaluations could be adopted from elsewhere, while the entire fission product (FP) database was adopted from JEFF-3.0.

The JEFF-3.1 evaluation for ${ }^{238} \mathrm{U}$ deserves special mention. Possible reasons for the underestimation of LEU criticality values have been identified as ${ }^{238} \mathrm{U}$ inelastic scattering and a slight overestimation of the ${ }^{238} \mathrm{U}$ capture cross section. A new ${ }^{238} \mathrm{U}$ evaluation has been undertaken, resulting in appreciably improved $k_{\text {eff }}$ values, especially for LWR lattices. Subgroup 22 of WPEC has contributed significantly to these improvements [15], and some of the benchmark results are described below. An evaluation example is given in figure 2. An eight-fold group representation was adopted for the delayed neutron data, as suggested by subgroup 6 of WPEC [16]. This representation has two advantages - longer lived, dominant precursors are explicitly represented, and conveniently the calculation has the same set of time constants for all fissioning isotopes.

Various experimental nuclear data activities have taken place during the development of JEFF-3.1. The n_TOF programme at CERN consisted of capture and fission crosssection measurements including isotopes of relevance to the thorium fuel cycle and several transuranic isotopes. A measurement programme at IRMM, Geel, covered mainly neutron data related to waste transmutation and other innovative concepts, as well as basic nuclear physics and standards data. 


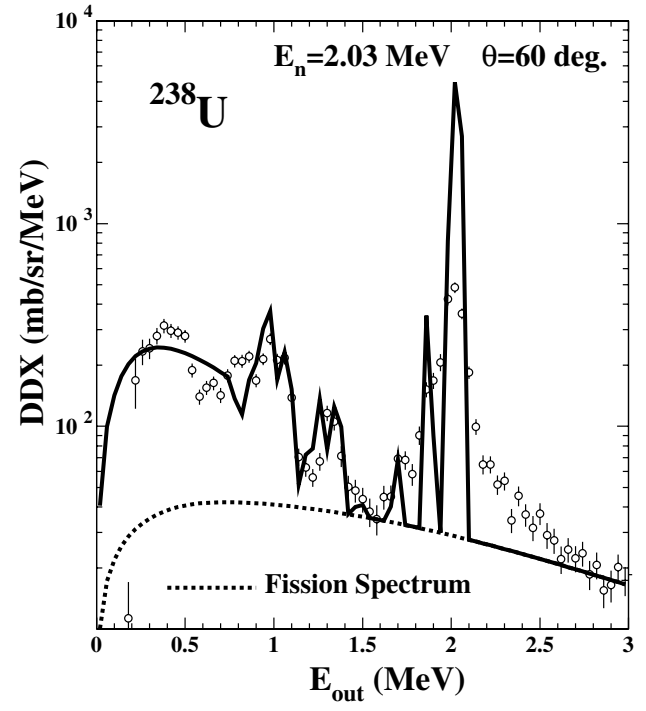

Fig. 2. Evaluated (calculated) double-differential ${ }^{238} U\left(n, n^{\prime}\right)$ spectrum at 2.03 MeV compared with experiment. Figure taken from [11].

In parallel, the TALYS nuclear modelling code developed by NRG and CEA [17] has been extensively used to produce new evaluations for JEFF-3.1.

A quality assurance procedure has been developed at the NEA Data Bank for the assembly, testing and loading of the JEFF-3.1 library into a database. Verifications performed at the loading stage include format and physics checks, processing using different versions of NJOY and graphical comparisons with other data libraries. Specific tools were also developed (e.g., JANIS data display and database loading programs), and existing programs were extended to enable more stringent consistency checks (e.g., FIZCON).

A final justification for releasing JEFF-3.1 in 2005 was a desire from the nuclear industry (in particular within France) to start validating the new JEFF library over a shortened timescale.

\subsection{Thermal scattering law library}

Revised thermal scattering law data have been produced for all important moderator and structural materials, and these files were included in JEFF-3.1 on the basis of nine evaluations: hydrogen bound in water, zirconium, polyethylene $\left(\mathrm{CH}_{2}\right)$ and $\mathrm{CaH}_{2}$, deuterium bound in $\mathrm{D}_{2} \mathrm{O},{ }^{9} \mathrm{Be}$, graphite, ${ }^{24} \mathrm{Mg}$ and calcium bound in $\mathrm{CaH}_{2}$. All files are new evaluations, except ${ }^{9} \mathrm{Be}$ and hydrogen in polyethylene, which are adopted from the JEFF-3.0 library. Many of the evaluations result from an IAEA data development project on thermal neutron scattering [18-21]. Calculations for a variety of temperatures were made with the LEAPR module of NJOY to obtain thermal scattering law data that are accurate over a wider range of energy and momentum transfer. Detailed comparisons with a significant number of measurements of differential and integral neutron cross sections and other relevant data have been performed in order to validate these files on a microscopic scale. The models used for these files are able to describe the experimental data reasonably well.

\subsection{Fission yield libraries}

The FP yield libraries JEFF-3.1/NFY\&SFY include independent and cumulative yields for the neutron-induced fission of ${ }^{232} \mathrm{Th},{ }^{233,234,235,236,238} \mathrm{U},{ }^{237,238} \mathrm{~Np},{ }^{238,239,240,241,242} \mathrm{Pu}$, ${ }^{241,242 m, 243} \mathrm{Am},{ }^{243,244,245} \mathrm{Cm}$ and the spontaneous fission of ${ }^{242,244} \mathrm{Cm}$ and ${ }^{252} \mathrm{Cf}$ [22]. These data are a development of UKFY3 [23]. The main changes with respect to earlier libraries include an extended experimental database, calculation of the cumulative yields using the JEFF-3.1 decay data, improved calculation of uncertainties in the yields, improved adjustment to the physical constraints and the inclusion of new ternary yield data for ${ }^{3} \mathrm{H}$ and ${ }^{4} \mathrm{He}$ [24]. Measurements performed at CEA have allowed an improvement of FP yield data in JEFF-3.1, particularly for the build-up of ${ }^{148} \mathrm{Nd}$ which is a main burnup indicator [25].

\subsection{Radioactive decay data library}

Radioactive decay data forms an integral part of the nuclear data requirements for nuclear applications. The decay data library JEFF-3.1/RDD contains 3852 nuclides from the neutron to ${ }^{272} \mathrm{Rg}$. The Decay Data sub-group of the JEFF Project decided that a completely new start should be made in the construction of this library compared with previous versions. NUBASE-2003 contains fundamental nuclear properties, and formed the basis for the identification of isotopes to be included in the decay data library [26]. Three evaluated libraries from within Europe were used to replace individual NUBASE files for almost 600 nuclei. Two libraries originated from the UK (UKPADD-6.4 and UKHEDD-2.4, sub-sets of refs. [27, 28]) and the third from the Decay Data Evaluation Project (DDEP) [29] overseen by the Laboratoire National Henri Becquerel (LNHB) at Saclay, France. Files were also selected from the ENSDF library that contains decay data identified with basic nuclear structure data [30]. Approximately 900 nuclei files were selected from ENSDF because they contain sufficient data to allow calculations of their energy balances to consistencies of better than $1 \%$. Recent enhancements that allow the storage of basic nuclear properties for stable nuclei have been adopted in this library (i.e., spin and parity). The isotopic abundances for all appropriate nuclei (taken from NUBASE-2003) have also been added to the library. Throughout the compilation process, the library has followed NEA QA procedures and has been checked with the standard set of ENDF Utility Codes, version 7. Furthermore, significant enhancements to the FIZCON code were made for verification of the recommended decay data.

\subsection{Activation library}

The JEFF-3.1/A activation data library in ENDF-6 format [31] is based on the European Activation File EAF-2003 [32]. The JEFF-3.1/A library contains 12617 excitation functions involving 774 different targets from ${ }^{1} \mathrm{H}$ to ${ }^{257} \mathrm{Fm}$, atomic numbers $\mathrm{Z}=1$ to 100 , in the energy range $10^{-5} \mathrm{eV}$ to $20 \mathrm{MeV}$. An uncertainty file is also provided that quantifies the degree of confidence placed in the data for each reaction channel. Cross section validation exercises against both experimental data and 
systematics enable a comprehensive assessment of the data. The SAFEPAQ-II software [33] is used to apply a series of adjustments to the original source data. A very important set of modifications concerns renormalization and branching using experimental or systematic data. A total of 3225 reactions ( $26 \%$ of all the reactions) have been changed in this manner, which was a challenging task as the source contains nonthreshold reactions with energy-dependent branching ratios.

\subsection{Special purpose proton library}

The special purpose proton library is a new addition to the JEFF files. It contains data for 26 evaluated isotopes. Data are based primarily on theoretical analyses with the TALYS code [17], in which the nuclear model parameters have been adjusted to reproduce the existing experimental data, after which dedicated formatting software produced the full ENDF6 data files. Valuable experimental data were provided by the EU FP5 HINDAS project for several materials of key importance to accelerator applications. This has resulted in files that provide a complete representation of the nuclear data needed for transport, damage, heating, radioactivity and shielding applications for protons with energies up to $200 \mathrm{MeV}$.

\section{Benchmarking}

Extensive, partly-automated benchmarking tests were undertaken to probe the quality of the new data file. These studies have included MCNP (NRG, SCK), TRIPOLI (CEA) [34] and APOLLO (CEA) criticality calculations for an unprecedented set of benchmarks. An example is given in figure 3, taken from refs. [35-37]. Current-day computer power will enable rapid revisions of the JEFF library to be quickly tested within this scheme. Additional validation is now possible by means of a Monte Carlo approach to the calculation of the effective delayed-neutron fractions [38], and improved thermal scattering law data [18-21]. A wider range of validation exercises is being performed [39-41] using different methods and codes to study various integral quantities. Apart from reactivity predictions in $\mathrm{UO}_{2}$-fuelled systems (CEA), JEFF-3.1 has exhibited improvements in isotopic inventory predictions as inferred from post irradiation examination data. All cases show improvements over the JEFF-3.0 library. However, some deficiencies do remain: core calculations with TRIPOLI4 [42] revealed an overestimation of $k_{\text {eff }}$ when simulating a MOX core, implying the need for an improved ${ }^{239} \mathrm{Pu}$ evaluation [43]. A detailed analysis of the MINERVE Oscillation experiment (OSMOSE) and PIE data for $\mathrm{UO}_{x}$ fuel led to the conclusion that the calculated reactivity worth is underestimated, indicating the possible adoption of a capture value for ${ }^{237} \mathrm{~Np}$ that is too low [44]. The MINERVE oscillation measurements also established the quality of the FP data of JEFF-3.1 [45].

The EFF project carries out integral experiments and develops calculational methods for fusion systems [46]. Test Blanket Modules (TBMs) are required for ITER, and they need to be designed in the near term. A major objective of the TBM tests is to demonstrate the tritium breeding performance of the breeder blanket concepts and to check and validate

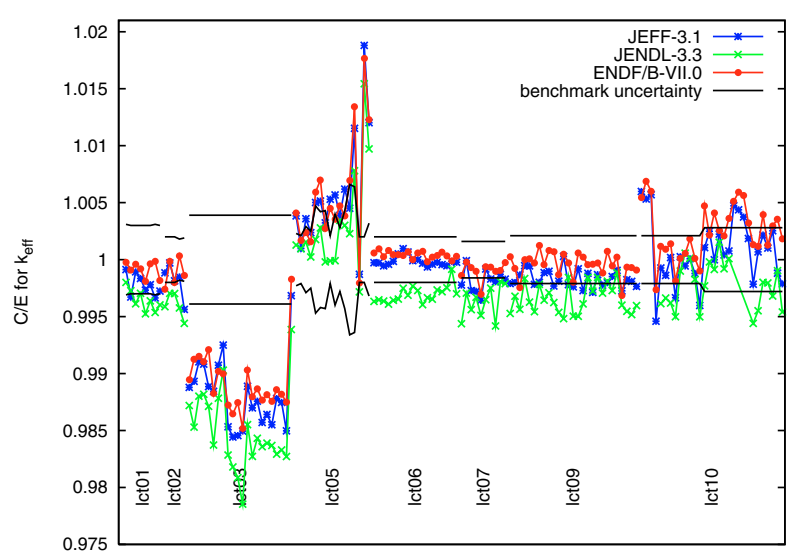

Fig. 3. Performance of JEFF-3.1 for various low enriched U thermal criticality benchmarks compared with other libraries.

the capability of the adopted data and neutronics codes to predict the nuclear responses with sufficiently good accuracy. Therefore, a facility has been built at ENEA to model the Helium Cooled Pebble Bed concept. Measurements of the tritium production rate and neutron/gamma flux by ENEA and TUD have been analysed with new and existing tools (FZK, HUJI, IJS) to check the adequacy of the nuclear data. Software tools have been developed to carry out sensitivity analyses within the neutronics codes (FZK, HUJI). Algorithms have been prepared to calculate variations in response due to changes in secondary angular distributions, and Monte Carlo track length estimators have been produced and implemented. These tools have subsequently been used in benchmark analyses. Future work includes an investigation of the derivation of covariances, evaluations at a range of energies and activation measurements of other suggested materials. Testing of JEFF3.1 for all materials with several existing $14-\mathrm{MeV}$ shielding benchmarks (LLNL, FNS, OKTAVIAN, FNG, TUD) has been completed [47].

Validation of the activation library against integral data has been performed by means of direct comparison with measurements of sample materials under fusion- and IFMIFrelevant [48] neutron spectra. Irradiations have been carried out at ENEA FNG, Sergiev Posad SNEG-13, JAERI FNS, FZK Isochron-cyclotron and NPI cyclotron, and comparisons made with integral $\mathrm{C} / \mathrm{E}$ data (ratio of a calculated library entry to the equivalent experimental value). The results of these benchmark exercises in concurrence with differential data have indicated where modifications to the data need to be made. Most of the measurements were carried out with materials relevant for fusion, resulting in small uncertainties for activities produced in the major nuclides, but larger uncertainties for activities produced in the minor nuclides and impurities. New methodologies for measuring the beta and gamma heating have also provided a large amount of data [49].

\section{Outlook: JEFF-3.2}

There is no firm timetable and completion target date for the preparation of the JEFF-3.2 library, but release is expected in 
2009. Until then, specific items to be addressed include the following:

- An extensive benchmarking report on JEFF-3.1, in which JEFF-3.1 will be tested against criticality, reactor burnup, shielding, activation, fission inventory and decay heat experiments, by means of a variety of Monte Carlo and deterministic computer codes.

- Inclusion of more covariance data: the availability and quality of covariance data is clearly inadequate. Various activities, such as WPEC subgroups 24 (covariances) and 26 (data needs for advanced reactors [50]) are addressing this matter, and the JEFF project should benefit from these initiatives.

- Revision and test of FPs: the JEFF project needs to exploit the FP evaluations undertaken as part of WPEC SG21 [51] and 23 and for ENDF/B-VII, for the selection of those data files that best reproduce the European integral measurements of FP capture.

- More emphasis on minor actinides: transmutation scenarios, GEN-IV systems and deep-burn designs require the accuracy of some minor actinide data to approach those of the major actinides.

- New photonuclear libraries, with special emphasis on actinides. Available libraries for actinides include data only up to $20 \mathrm{MeV}$. New evaluations for ${ }^{232} \mathrm{Th},{ }^{235,238} \mathrm{U}$, ${ }^{237} \mathrm{~Np}$ and ${ }^{239} \mathrm{Pu}$ will be extended to $130 \mathrm{MeV}$ and recent measurements of delayed neutron data will be included.

- New proton and deuteron libraries: these data will be primarily based on available models.

- Adoption of EAF-2007 as new activation library: includes an energy extension to $60 \mathrm{MeV}$, which is required for the IFMIF fusion programme [52].

- Update of the decay data and fission yield libraries: inconsistencies will be corrected and additional spectral data will be included for specific radionuclides.

- Thermal scattering law data will undergo further testing.

- More complete gamma production data: presence of gamma production data in all libraries is still rather random - a more systematic approach to the production of gamma data is needed, and this issue will be taken up in future releases of JEFF.

- TALYS-1.0 will be used in many future evaluations.

- NJOY extensions required for JEFF processing: a special NJOY users group [53] has been launched inside the JEFF project.

- CONRAD: new, modern modular software developed by CEA, Cadarache, for the analysis of nuclear data [54] will provide a natural interface between the resolved and unresolved resonance region and fast neutron range in neutron data evaluations.

Specific neutron evaluations that will be renewed or updated:

- Revision and validation of ${ }^{235,238} U$ to solve remaining deficiencies, especially for fast HEU systems.

- New evaluation for ${ }^{239} \mathrm{Pu}$ to improve MOX analyses.

- New evaluations of $\mathrm{Cr}, \mathrm{Mn}$, Ta and $\mathrm{W}$ isotopes are available from the EFF fusion project.

- Revised evaluation for Pb, Bi and Am using IRMM, Geel, measurements and TALYS.
- New evaluation for ${ }^{232} \mathrm{Th},{ }^{233} \mathrm{~Pa}$ based on $\mathrm{n}_{-}$TOF/IRMM measurements - these data files are the results of an IAEA coordinated research project on the Th fuel cycle [55] and have also been adopted in ENDF/B-VII.

- New evaluations for ${ }^{2} \mathrm{H},{ }^{151} \mathrm{Sm},{ }^{237} \mathrm{~Np}, \mathrm{Zr}$ and $\mathrm{Hf}$-isotopes.

We foresee validation and benchmarking activities on the following:

- Criticality studies by means of Monte Carlo: large-scale validation of the ICSBEP criticality data is planned that will be based on MCNP and TRIPOLI, and wellautomated procedures within the JEFF project (NRG, CEA/Cadarache, SCK, VTT, etc.).

- Integral experiments with deterministic methods: codes such as ERANOS [56] and SCALE are being used to test the JEFF-3.1 library for fuel inventory, reactivity variation, and FP integral experiments.

- 14-MeV shielding benchmarks: LLNL, FNS, OKTAVIAN, FNG, TUD benchmarks will be tested for new JEFF library releases, especially on the basis of the Monte Carlo codes (FZK, NRG, SCK).

- Activation benchmarks: follow-up of the fusion activation benchmarks as already mentioned in section 3 .

- Decay heat benchmarks.

- IRPHE benchmarks on larger reactor systems.

\section{Conclusions}

The JEFF-3.1 nuclear data library was released in May 2005. Concerted efforts have been expended on all of the general and special purpose libraries to achieve overall improvements over a range of simulations for nuclear technology applications. Remaining deficiencies pose new challenges to and incentives for the further development of JEFF. Proposed future objectives have been listed in this paper, and need to be realised in order to meet users' demands and needs.

\section{References}

1. The JEFF-3.1 Nuclear Data Library, JEFF Report 21, NEA/OECD No. 6190, 2006, ISBN 92-64-02314-3, http://www.nea.fr/html/dbdata/projects/nds_jef.htm.

2. A.J. Koning, M.C. Duijvestijn, New nuclear data evaluations for Ca and Sc isotopes, J. Nucl. Sci. Technol. (to be published).

3. S. Tagesen, H. Vonach, Status of the evaluation of all Ti isotopes, EFFDOC-879 (2002).

4. M.C. Duijvestijn, A.J. Koning, New intermediate-energy nuclear data libraries for Fe, Ann. Nucl. Energy 33, 1196 (2006).

5. A.J. Koning, M.C. Duijvestijn, New nuclear data evaluations for Ge isotopes, Nucl. Instrum. Meth. B 248, 197 (2006).

6. F. Gunsing and O. Serot, Progress on Tc-99 evaluation, JEF/DOC-1054 (2004).

7. E. Dupont et al., Neutron data evaluation and validation of Rhodium-103, in Proc. Int. Conf. Nucl. Data for Sci. Techn. ND2004, AIP 769, 2004, Santa Fe, USA, eds. R.C. Haight et al. (2005), p. 95.

8. E. Dupont et al., Neutron Data evaluation of I-127 and I-129 isotopes, JEF/DOC-1076 (2005). 
9. G. Noguere, Low neutron cross sections of the Hf isotopes, JEF/DOC-1077 (2005).

10. A.J. Koning et al., New nuclear data libraries for $\mathrm{Pb}$ and $\mathrm{Bi}$ and their impact on ADS design, Nucl. Sci. Eng. (to be published).

11. M.J. López Jiménez et al., Triple-humped fission barrier model for a new ${ }^{238}$ U neutron cross-section evaluation and first validations, Ann. Nucl. Energy 32, 195 (2006).

12. A. Trkov et al., Revisiting the ${ }^{238} U$ thermal capture cross section and gamma-ray emission probabilities from ${ }^{239} \mathrm{~Np}$ decay, Nucl. Sci. Eng. 150, 336 (2005).

13. A. Courcelle et al., Evaluation of ${ }^{238} U$ Resonance Parameters from 0 to $20 \mathrm{keV}$, Proc. Int. Conf. Nucl. Data for Sci. Techn. - ND2004, AIP 769, 2004, Santa Fe, USA, eds. R.C. Haight et al., (2005), p. 276.

14. O. Bouland, D. Bernard, Revised evaluation of the ${ }^{241}$ Am isotope, JEF/DOC-1086 (2005).

15. A. Courcelle et al., Nuclear data for improved LEU-LWR reactivity predictions, NEA/WPEC-22, OECD (2006).

16. A. D'Angelo et al., Delayed neutron data for the major actinides, NEA/WPEC-6, OECD (2002).

17. A.J. Koning et al., TALYS-1.0 (these proceedings).

18. See webpage: http://www-nds.iaea.org/indltsl/.

19. M. Mattes, J. Keinert, Thermal neutron scattering data for the moderator materials $\mathrm{H}_{2} \mathrm{O}, \mathrm{D}_{2} \mathrm{O}$ and $\mathrm{ZrH}_{x}$ in ENDF-6 format and as ACE library for $M C N P(X)$ codes, IAEA Report INDC(NDS)0470 (2005).

20. M. Mattes, J. Keinert, Status of thermal neutron scattering data for graphite, IAEA Report INDC(NDS)-0475 (2005).

21. A. Trkov, M. Mattes, On the thermal scattering law data for reactor lattice calculations, Int. Conf. on Nuclear Energy for New Europe 2004, 6-9 September 2004, Portoroz, Slovenia.

22. R.W. Mills, Initial benchmarking of the JEFF3.1 fission yields and decay data, JEF/DOC-1070 (2005).

23. R.W. Mills, Fission product yield evaluation, Ph.D. thesis, The University of Birmingham, UK (1995).

24. O. Serot et al., New results on helium and tritium gas production from ternary fission, in Proc. Int. Conf. Nucl. Data for Sci. Techn. - ND2004, AIP 769, 2004, Santa Fe, USA, eds. R.C. Haight et al. (2005), p. 857.

25. O. Serot et al., Review of the most reactor relevant cumulated $F P$ yields, JEF/DOC-1055 (2004).

26. G. Audi et al., The NUBASE evaluation of nuclear and decay properties, Nucl. Phys. A 729, 3 (2003).

27. A.L. Nichols, R.J. Perry, Activation product decay data: UKPADD6.7, Serco report SA/NST/18923/W001, Issue 1; UKNSF(2007)P212, JEF/DOC-1165 (2007).

28. A.L. Nichols, R.J. Perry, Heavy element and actinide decay data: UKHEDD2.5, Serco report SA/NST/18923/W002, Issue 1; UKNSF(2007)P213, JEF/DOC-1166 (2007).

29. M.-M. Bé et al., Table of Radionuclides, Monographie BIPM-5, ISSN 92-822-2204-7 (set), ISBN 92-822-22187 (Vol. 3) et ISBN 92-822-2219-5 (CD), CEA/LNELNHB, 91191 Gif-sur-Yvette, France and BIPM, Pavillon de Breteuil, 92312 Sèvres, France. See data at http://www.nucleide.org/DDEP_WG/DDEPdata.htm.

30. See webpages http://nds121.iaea.org/ensdf/index.jsp and http://www.nndc.bnl.gov/ensdf/.

31. J.-Ch. Sublet et al., The JEFF-3.1/A Neutron Activation File New Features, Validation and Usage, in Proc. Int. Conf. Nucl. Data for Sci. Techn. - ND2004, AIP 769, 2004, Santa Fe, USA, eds. R.C. Haight et al. (2005), p. 203.
32. R.A. Forrest et al., The European Activation File: EASY-2003 cross section library, UKAEA FUS 486 (2003). All EASY documents available from http://www.fusion.org.uk/easy2007.

33. R.A. Forrest, SAFEPAQ-II, a new tool for the production of activation data libraries, Fus. Eng. Des. 54, 387 (2001).

34. C. Jouanne, J.-Ch. Sublet, TRIPOLI-4.4 JEFF-3.1 Based Libraries, CEA-R6125 (2006).

35. S.C. van der Marck, Criticality safety benchmark calculations with MCNP-4C3 using JEFF-3.1 nuclear data, NRG Report 21616/05.69456/P, JEF/DOC-1107 (2005).

36. S.C. van der Marck and A. Hogenbirk, Criticality results for many benchmark cases - The releases JEFF-3.0, ENDF$B / V I .8$, JENDL-3.3, ENDF-B/VII-prelim and BRC, JEF/DOC974 (2003).

37. S.C. van der Marck, Benchmarking ENDF/B-VII.O, Nucl. Data Sheets 107, 3061 (2006).

38. S.C. van der Marck, $\beta_{\text {eff }}$ calculations using JEFF-3.1 nuclear data, NRG Report 21616/05.69454/P, JEF/DOC-1105 (2005).

39. I. Duhamel, Benchmarking of JEFF3.1 evaluation against critical integral experiments (these proceedings).

40. W. Zwermann, Monte Carlo calculations with nuclear point data based on JEF-2.2 and JEFF-3.1 for the VENUS-7 critical benchmarks (these proceedings).

41. M. Pescarini, ENEA-Bologna production and testing of JEFF3.1 multi-group cross section libraries for nuclear fission applications (these proceedings).

42. O. Litaize et al., JEFF-3.1 LWR-reactivity qualification from EOLE experiments, JEF/DOC-1143 (2006).

43. D. Bernard, A. Santamarina, Improvements of the 239Pu evaluation for JEFF3, JEF/DOC-1158 (2006).

44. D. Bernard, A. Santamarina, Qualification of ${ }^{237} N p$ cross sections. Required modifications in JEFF3.1, JEF/DOC-1144 (2006).

45. A. Courcelle et al., Experimental Validation of Main Fission Products and Actinide Nuclear Data. Improvements in JEFF-3, Proc. of Int. Conf. PHYSOR2002, Seoul, 2002.

46. P. Batistoni et al., Neutronics design and supporting experimental activities in the EU, Fusion Eng. Des. 81, 1169 (2006).

47. S.C. van der Marck, Shielding benchmark calculations with MCNP-4C3 using JEFF-3.1 nuclear data, NRG Report 21616/05.69455/P, JEF/DOC-1106 (2005).

48. R.A. Forrest et al., Validation of EASY-2005 using integral measurements, Report UKAEA FUS 526, 2006.

49. M. Pillon et al., Measurements of decay heat and validation of the European activation code system for fusion power plant applications, Fusion Eng. Des. 63, 101 (2002).

50. M. Salvatores, Nuclear data needs for advanced reactor systems. A NEA Nuclear Science Committee initiative (these proceedings).

51. P. Oblozinsky et al., Assessment of neutron cross-section evaluations for the bulk of fission products, NEA/WPEC-21, OECD (2005).

52. R.A. Forrest, J. Kopecky, EASY-2007: a new generation of activation modelling including neutron-, proton- and deuteroninduced reactions (these proceedings).

53. Unified NJOY updates can be found at http://www.nea.fr/html/dbprog/njoy-links.html.

54. C. De Saint Jean, Presentation of CONRAD, a new software for nuclear data analysis, JEF/DOC-1142 (2006).

55. Evaluated nuclear data for Thorium-Uranium fuel cycle, IAEA CRP, project page: http://www-nds.iaea.org/Th-U/.

56. J. Tommasi, Present status of JEFF-3.1 benchmarking with ERANOS-2.0, JEF/DOC-1114 (2005). 